\title{
Anthropometric Characteristics and Mineral Distribution and Contamination in Artisanal Small-scale Gold Mining Site of Ciguha in Gunung Pongkor, Bogor
}

Haryoto Kusnoputranto ${ }^{1}$, Jonatan Oktoris Simanjuntak ${ }^{2}$, Nila Puspita Sari ${ }^{3}$, Bambang Wispriyono ${ }^{4}$ and Abdur $\operatorname{Rahman}^{5, *}$

${ }^{1}$ Department of Environmental Health, Faculty of Public Health, Universitas Indonesia, Depok 16424, and Graduate School of Environment, Universitas Indonesia, Salemba 4, Jakarta, Indonesia

${ }^{2}$ Department of Environmental Health, Faculty of Public Health Universitas Indonesia, Depok 16424, Indonesia

${ }^{3}$ Department of Environmental Health, Faculty of Public Health Universitas Indonesia, Depok 16424, Indonesia

${ }^{4}$ Department of Environmental Health, Faculty of Public Health Universitas Indonesia, Depok 16424, Indonesia

${ }^{5}$ Department of Environmental Health, Faculty of Public Health Universitas Indonesia, Depok 16424, Indonesia

*Corresponding author's email: abd.rahman [AT] ui.ac.id

\begin{abstract}
Ciguha in Gunung Pongkor, Bogor, had been an artisanal and small-scale gold mining site (ASGM) since 1998 where amalgamations were used intensively leading to pollution in water, soil, and foodstuffs. The present study assessed distribution and contamination level (CL) of minerals in a total of 69 environmental samples consisting of drinking water $(n=12)$, rice $(n=13)$, vegetable $(n=15)$, fruits $(n=5)$, fish $(n=14)$, and soil $(n=10)$. Chromium, iron, and manganese representing trace essential elements but toxic at high level, mercury representing heavy metal, arsenic representing toxic metalloid, and selenium representing essential metalloid which toxic at high level, were analyzed as mineral contaminants. Meanwhile, a total of 101 Ciguha residents consisting of 60 adults, 15 teenagers, and 26 school aged children were involved to charaterize athropometric exposure factors. The results shows that mercury has polluted soil, kangkung, thai squash, cassava leaves, and rice with contamination level (CL) of 604.43, $8.15,6.01,4.14$, and 2.76 folds, respectively, while chromium has only polluted thai squash with CL of 1.18 folds. Amazingly, mercury distribution was only in the third position after iron and manganese, while the most distributed mineral was iron and the least was selenium. In overall environmental matrices, the detection frequencies of iron, manganese, mercury, chromium, arsenic, and selenium were 94.2, 56.2, 52.2, 47.8, 21.7, and 15.9\%, respectively. In conclusion, mercury was the most critical contaminant in ASGM site of Ciguha that has heavily polluted soil and grown vegetables, but the most distributed mineral was iron. Toxicologically, only mercury and chromium are important while arsenic, iron, manganese, and selenium are of less concern since the $C L<1$.
\end{abstract}

Keywords - Artisanal and small-scale gold mining (ASGM), contamination level, mineral, ciguha

\section{INTRODUCTION}

In the last two decades, Gunung Pongkor in Bogor Regency, West Java, has become an attractive area due to high mineral accumulation. It has gold-silver deposit of about 1.3 million oz that can be exploited until 2019. PT Aneka Tambang Tbk, a state-own company known better as Antam, has exploited the gold deposit since 1992 in 14,940-acres concession area including community residents, agriculture field, and protected forest. Using cyanidation for gold extraction, Antam produces 1,500 to $2,000 \mathrm{~kg}$ gold annually [1].

Antam gold mining has attracted thousands artisanal miners from out side Gunung Pongkor to mine gold using simple, unsafe extraction techniques [2]. The artisanal miners, or gurandil in local language, extract gold by intensive amalgamation. All the processes are conducted in the residential areas in improper buildings or even inside the houses without adequate ventilation or exhaust. There are no appropriate control measures at all in these extraction processes. 
Improper gold extraction in artisanal and small-scale gold mining (ASGM) site by gurandils raises public concerns about mercury and other toxic mineral contamination in environmental media. Some unwanted metals, metalloids, and nonmetals may release to the environment during soil crushing, amalgamation, crude gold recovery, and tailing disposal, resulting in pollution in air, water, soil, and grown foods. Previous study showed that surface water and groundwater in Gunung Pongkor contained high level of heavy metals (such as mercury and chromium) and metalloid (such arsenic) [3].

So far, studies in Gunung Pongkor mostly focused on mineral geology and environmental pollution, particularly on mercury [3, 4]. But, an environmental health risk assessment had been conducted in Bantar Karet, Cisarua, and Malasari, the three villages in Ring-1 of Antam mining site [5]. It analyzed transition metals, heavy metals, metalloids, and anionic contaminants in drinking water and grown foods. It revealed that arsenic, cyanide, chromium, fluoride, and nitrite in drinking water exceeded the national standard (Permenkes No. 492/2010) by 1.2 (As) to 700 (cyanide) folds excess. In foods, arsenic was detected in rice, vegetables, cassava, banana, and fish, while lead was detected in rice, vegetables, cassava, and banana. Meanwhile, cadmium was detected in fish and mercury in rice. Employing anthropometric exposure factors (body weight, consumption rate, and contact time rate, generated from 200 local villagers), exposures to those minerals from drinking water has brought about unacceptable noncarcinogenic health risk estimates of cyanide, arsenic, fluoride, chromium, nitrite, and mercury with risk quotient (RQ) of 86.02, 41.33, 26.34, 3.37, and 1.85, respectively. Excess cancer risk (ECR) estimates from exposure to arsenic in drinking water, vegetables, cassava, banana, and fish were also unacceptable with ECR of 2.69E-4, 2.19E-3, 1.06E-4, 8.85E-5, and 6.6E-3, respectively.

High contamination level (CL) of minerals is not always resulted in high health risks and vice versa because health risk depends on chronic intake, while the chronic intake depends also on parameters other than concentration such as contact time and consumption rates. For example, in Gunung Pongkor arsenic has the lowest CL among six minerals in drinking water, but its overall RQ is in the second top after cyanide due to contribution of ingestion of arsenic-containing foods. Similarly, mercury has low CL in drinking water, but food ingestion makes its RQ unacceptable [5].

It is therefore important to assess distribution of mineral contaminants in all environmental exposure media that may contribute to the overall intakes. Local foods grown in ASGM site are critical, since the foodstuffs are product of complex physical, chemical, and biological processes of essential and beneficial chemical elements in multimedia environment. In order to develop control measures to manage health risks from exposure to minerals in ASGM site, a public health risk assessment (PHRA) has been conducted recently in Ciguha hamlet in Bantar Karet village. It was intended to derive such safe exposure model that the daily intake of each mineral does not exceed its toxic level and, for essential mineral, meets its essentiality doses. It analyzed six minerals representing heavy metals, trace essential elements but toxic at high level, toxic metalloid, and essential metalloid in drinking water, foods, and soil. Health risks, estimated from exposure to these minerals, were avaluated in line with disease signs and symptoms and community concerns that may reflect physical, emotional, mental, and spiritual health associated with mineral intakes. Finaly, the present PHRA derived safe exposure level model and safe consumption guideline for local residents. The present article repots the first part of the PHRA study regarding anthropometric characteristics of the exposed population and distribution and CL of arsenic, chromium, iron, manganese, mercury, and selenium in drinking water, foodstuffs, and soils.

\section{METHODS}

\subsection{Site Description and Demography}

Gunung Pongkor in Bogor Regency, where Ciguha hamlet is located, is a concession area of Antam gold mining site. Administratively, Antam gold mining site belongs to 3 sub-districts i.e. Nanggung (70\%), Leuwiliang (20\%), and Cigudeg (10\%). Its area spreads out from $106^{\circ} 31^{\prime}$ to $106^{\circ} 31^{\prime}$ East Longitude and from $06^{\circ} 35^{\prime}$ to $6^{\circ} 42^{\prime}$ South Latitude with elevation ranging from 375 to $850 \mathrm{~m}$ above sea level. Ciguha is an important hamlet in Bantar Karet village in Nanggung Sub-District, because its area is overlapped with the Antam site. Bantar Karet is inhabitted by 10,329 people (all ages, 2015 data) with 2,935 household heads and spread out in 38 Household Clusters (Rukun Tetangga, RT). Ciguha has been existed as rural settlement for more than 20 years before Antam established its gold mining site. Currently, Ciguha has 181 households in 2 RTs. Ciguha is the closest hamlet to Ring-1 Antam site and has highest number of ASGM miners or gurandils.

\subsection{Population and Samples}

Household was assigned as unit of analysis from which samples of human subjects and exposure media were to be collected. Sample size was determined by proportion $(\mathrm{P})$ estimate for one population with specified absolute precision (d) [6]. Using 0.032 for $P$ (indication of keratosis-like disease found in the previous study in Gunung Pongkor [5]) and 0.05 for $d$, with $95 \%$ confident interval the required sample was 60 households. From these households, 60 adults, 60 teenagers, 60 school age children, 15 samples of foodtuffs items (rice, vegetables, fruits, fishes), and 15 soil samples (where samples of vegetables and /or fruits were grown) were to be selected randomly.

\subsection{Mineral Analysis}


The present study selected six minerals (.e. chromium, iron, and manganese representing trace essential elements but toxic at high level, mercury representing heavy metal, arsenic representing toxic metalloid, and selenium representing essential metalloid but toxic at high level) as ASGM contaminants. Samples of foodstuffs were prepared for mineral analysis using techniques as decribed elsewhere [7] and are summarized as follows. Rice, vegetables, skin-off fruits, and fillet fishes were washed by clean water then by distilled water, and dried in open air. The dried samples were crushed in mortar or blended using stailess knives. Apprimately $10 \mathrm{~g}$ of each crushed sample was placed in $25-\mathrm{mL}$ erlenmeyer. In a fume cupboad, $5 \mathrm{~mL}$ of concentrated nitric acid were added into the prepared sample, mixed thouroughy, and $5 \mathrm{~mL}$ concentrated hydrochloric acid were then added dropwise. After standing overnight for cool reaction, the reaction mixture was heated at 80 to $95{ }^{\circ} \mathrm{C}$ for 2 hours. Distilled water was re-added and the mixture was re-heated repeatedly until clear, colorless solution was obtained with $\mathrm{pH} \geq 4$. After cooling, the solution was filtered off through No. 41 Whatman paper. The filtrate was tranfered into $25-\mathrm{mL}$ volumetric flask, then diluted by distilled quantitatively. Chromium, iron, and manganese in the solution were determined by UV-vis spectrophometer, while arsenic, mercury, and selenium are determined by Atomic Absorption Spectrophometer. Concentrations were expresses in mg mineral per $\mathrm{kg}$ dry weight of foodstuff.

\subsection{Anthropmetric Exposure Factor Characteristics}

Anthropometric exposure factors required for estimating dietary intake of essential minerals were body weight, consumption rate (drinking water and foods), and activity pattern (exposure time and duration). Body weight was determined by weighing the subjects using pre-calibrated scale in $0.1 \mathrm{~kg}$ precision. Drinking water comsumption rate was determined by cup method as described elsewhere [8] in $10 \mathrm{~mL}$ precision, while food consumption rates were determined for foodstuffs (not meals or mixtures) by 24-HDR. By cup method, subjects were asked to apporximate how many cups (of household size) of water they drink daily. The daily drinking water consumption rate was determined by multiplying the number of cups by cup volume. Food items in the diet, classified into grain or carbohydrate, vegetable, fruit, and fish, were recalled from the last four concecutive meals (yeseterday breakfast, yesterday lunch, yesterday dinner, and today breakfast). Quantity of each food item consumed was determined by weighing the amount of real foodstuffs (not food model) as shown by the subjects in $1 \mathrm{~g}$ precision, then convert to weight of edible part [9]. Foodstuff samples were those which meet the following inclusive criteria: the most frequently consumed daily compared to other foodsuffs within the same food group, consumed at least once a week, and available in raw state (uncooked).

\subsection{Contamination Level}

CL criterion is employed to determine pollution order of magnitude of particular contaminant in specified environmental media. CL of a mineral was determined as ratio of detected concentration of the mineral in a specified environmental medium to its reference value (RV). The RV can be either the environmental standard, guideline, background level, or limit value derived from toxicity data. The RV each drinking water minerals may refer to the Ministry of Health Regulation No. 492 of 2010 (known better as Permenkes 492/2010), but in the present study the drinking water RV was assigned as drinking water equivalent level (DWEL) [10], while the foodstuff RV was assigned as food equivalent level (FEL). Both DWEL and FEL were derived from reference dose (RfD, $\mathrm{mg} / \mathrm{kg} / \mathrm{day}$ ) by inputing body weight $\left(W_{\mathrm{B}}, \mathrm{kg}\right)$ and consumption rate $(R, \mathrm{~L} /$ day for drinking water, $\mathrm{kg} /$ day for foodstuffs $)$ as expressed in Eq. (1). For soil minerals, the RV used guideline values either from other countries, background level, or data from various studies published elsewhere.

$$
R V=\frac{R f D \times W_{\mathrm{B}}}{R}
$$

\section{RESULTS}

\subsection{Anthropmetric Exposure Factor Characteristics}

It was designed to survey 180 subjects from 60 selected households, but only adults residents met the required number of 60 individuals. Due to limited permit to enter to Ciguha, there were only 15 teenagers and 26 school aged children available to survey. Anthropometric exposure factor charateristics of Ciguha residents of three age groups are summarized in Table 1.

Tabel 1: Statistical Summary of Anthropometric Exposure Factors

\begin{tabular}{lllll}
\hline Exposure factors & Range & Median & Mean \pm SD & Distribution \\
\hline Anthropometry & & & & \\
$\quad$ Body weight $(\mathrm{kg})$ & & & & \\
$\quad$ Adults & $35.6-78$ & 52.3 & $53.5 \pm 7.1$ & Normal \\
$\quad$ Teenagers & $39.9-51.7$ & 47.3 & $47.1 \pm 3.4$ & Normal \\
$\quad$ School age children & $21.3-36.8$ & 29.9 & $29.5 \pm 4.2$ & Normal \\
Body height $(\mathrm{cm})$ & & & & \\
\hline
\end{tabular}




\begin{tabular}{|c|c|c|c|c|}
\hline Exposure factors & Range & Median & Mean \pm SD & Distribution \\
\hline Adults & $145-171$ & 160 & $158 \pm 7$ & Normal \\
\hline Teenagers & $149-166$ & 160 & $159 \pm 5$ & Normal \\
\hline School age children & $113-160$ & 133 & $133 \pm 1$ & Normal \\
\hline \multicolumn{5}{|l|}{ Age (year) } \\
\hline Adults & $18-85$ & 33 & $35 \pm 13$ & Normal \\
\hline Teenagers & $12-17$ & 14 & $15 \pm 1.5$ & Normal \\
\hline School age children & $6-11$ & 9.1 & $9 \pm 1.5$ & Normal \\
\hline \multicolumn{5}{|l|}{ Activity } \\
\hline $\begin{array}{l}\text { Exposure frequency }\left(f_{\mathrm{E}} \text {, day/year), }\right. \\
\text { all ages (life span) }\end{array}$ & $317-365$ & 363 & $356 \pm 14$ & Not Normal \\
\hline \multicolumn{5}{|l|}{ Exposure duration $\left(D_{\mathrm{E}}\right.$, year $)$} \\
\hline Adults & $2.99-80$ & 20 & $25 \pm 17$ & Not Normal \\
\hline Teenagers & 6 & - & - & Not applicable \\
\hline School age children & 6 & - & - & Not applicable \\
\hline
\end{tabular}

From the selected 60 households, the 24-HDR survey identified the frequent consumed local foods i.e. rice as the only source of carbohyrate, 6 local fruits (banana, orange, papaya, mango, guava, and snake-skin fruit or salak), 2 nonlocal fruits (apple and melon), 6 local vegetables (cassava leaves, stringbean, thai squash, kangkung (an edible water hyacinth), long bean, and spinach), fresh water fish (golden fish, or common carp), and 7 nonlocal protein foods (egg, tofu, salted marine fish, tempeh, chicken, sardines, and meat). Statistical summary of food consumption rates among Ciguha redidents is presented in Table 2.

Tabel 2: Statistical Summary of Consumption Rate

\begin{tabular}{|c|c|c|c|c|}
\hline Water and Foodstuffs & Range & Median & Mean \pm SD & Distribution \\
\hline \multicolumn{5}{|l|}{ Driking water (L/day) } \\
\hline Adults & $0.4-8.0$ & 1.0 & $1.262 \pm 1.125$ & Not Normal \\
\hline Teenagers & $0.4-4$ & 0.9 & $1.123 \pm 0.844$ & Not Normal \\
\hline School age children & $0.2-2$ & 0.5 & $0.609 \pm 0.5$ & Not Normal \\
\hline \multicolumn{5}{|l|}{ Rice/grain (g/day) } \\
\hline Adults & $200-900$ & 400 & $445 \pm 171$ & Normal \\
\hline Teenagers & $200-900$ & 400 & $470 \pm 183$ & Normal \\
\hline School age children & $100-400$ & 200 & $226 \pm 76$ & Normal \\
\hline \multicolumn{5}{|l|}{ Vegetable (g/day) } \\
\hline \multicolumn{5}{|l|}{ Adults } \\
\hline Cassava leaves $(n=50)$ & $6.67-300$ & 28.57 & $45.98 \pm 52.27$ & Not normal \\
\hline Kangkung $(\mathrm{n}=24)$ & $6.67-200$ & 14.29 & $38.07 \pm 43.27$ & Not normal \\
\hline Thai squash $(n=22)$ & $14.29-300$ & 57.14 & $76.08 \pm 72.67$ & Not normal \\
\hline Spinach $(n=20)$ & $6.67-200$ & 14.29 & $39.98 \pm 46.91$ & Not normal \\
\hline Long been $(n=17)$ & $3.33-100$ & 14.29 & $31.71 \pm 28.69$ & Not normal \\
\hline String bean $(\mathrm{n}=13)$ & $14.29-142.86$ & 35,71 & $62.64 \pm 47.48$ & Normal \\
\hline \multicolumn{5}{|l|}{ Teenagers } \\
\hline Cassava leaves $(\mathrm{n}=11)$ & $14.29-100$ & 14.29 & $35.06 \pm 33.42$ & Not normal \\
\hline Thai squash $(\mathrm{n}=8)$ & $14.29-100$ & 15.48 & $43.15 \pm 42.29$ & Normal \\
\hline Spinach $(\mathrm{n}=8)$ & $6.67-100$ & 14.29 & $31.19 \pm 31.98$ & Not normal \\
\hline Kangkung $(\mathrm{n}=5)$ & $6.67-100$ & 14.29 & $38.48 \pm 39.74$ & Normal \\
\hline String bean $(n=3)$ & $14.29-100$ & 28.57 & $47.62 \pm 45.92$ & Normal \\
\hline Long been $(n=3)$ & $14.29-100$ & 14.29 & $42.86 \pm 49.49$ & Normal \\
\hline \multicolumn{5}{|l|}{ School aged children } \\
\hline Cassava leaves $(n=20)$ & $3.33-100$ & 14.29 & $23.38 \pm 24.25$ & Not normal \\
\hline String bean $(n=9)$ & $14.29-50$ & 14.29 & $21.43 \pm 12.37$ & Not normal \\
\hline Spinach $(n=9)$ & $3.57-100$ & 7.14 & $23.41 \pm 30.36$ & Not normal \\
\hline Kangkung $(\mathrm{n}=9)$ & $3.57-100$ & 7.14 & $22.62 \pm 30.51$ & Not normal \\
\hline Long bean $(n=9)$ & $7.14-25$ & 7.14 & $10.71-6.19$ & Not normal \\
\hline Thai squash $(\mathrm{n}=8)$ & $7.14-71.43$ & 19.64 & $32.59 \pm 27.48$ & Normal \\
\hline \multicolumn{5}{|l|}{ Fruit (g/day) } \\
\hline \multicolumn{5}{|l|}{ Adults } \\
\hline Banana $(\mathrm{n}=38)$ & $1.50-450$ & 12.87 & $31.60 \pm 75.66$ & Not normal \\
\hline Orange $(n=23)$ & $3.67-440$ & 23.571 & $46.004 \pm 89.43$ & Not normal \\
\hline Papaya $(\mathrm{n}=11)$ & $3.67-125.71$ & 15.71 & $46.05 \pm 42.05$ & Normal \\
\hline Guava $(n=6)$ & $10-57.14$ & 20.79 & $24.55 \pm 18.17$ & Normal \\
\hline
\end{tabular}




\begin{tabular}{|c|c|c|c|c|}
\hline Water and Foodstuffs & Range & Median & Mean $\pm \mathrm{SD}$ & Distribution \\
\hline Salak $(n=6)$ & $3.25-18.57$ & 7.04 & $8.85 \pm 6.39$ & Normal \\
\hline Mango $(n=6)$ & $3-38.57$ & 17.36 & $20.14 \pm 16.3$ & Normal \\
\hline Melon $(n=2)$ & $38-54.29$ & 46.14 & $46.14 \pm 11.51$ & Not applicable \\
\hline Apple $(\mathrm{n}=1)$ & 24.29 & 24.29 & 24.29 & Not applicable \\
\hline \multicolumn{5}{|l|}{ Teenagers } \\
\hline Orange $(n=9)$ & $3.67-110$ & 15.71 & $29.51 \pm 33.92$ & Not normal \\
\hline Banana $(\mathrm{n}=8)$ & $1.5-160.71$ & 28.93 & $45.99 \pm 55.17$ & Not normal \\
\hline Salak $(n=5)$ & $3.25-13.93$ & 4.33 & $6.90 \pm 4.76$ & Normal \\
\hline Mango $(\mathrm{n}=3)$ & $6-9$ & 6 & $7 \pm 1.73$ & Normal \\
\hline Papaya $(n=2)$ & $15.71-62.86$ & 39.29 & $39.29 \pm 33.33$ & Not applicable \\
\hline Guava $(\mathrm{n}=2)$ & $10-28.57$ & 19.29 & $19.29 \pm 13.13$ & Not applicable \\
\hline \multicolumn{5}{|l|}{ School age children } \\
\hline Banana $(n=15)$ & $0.75-45$ & 3.21 & $8.92 \pm 11.56$ & Not normal \\
\hline Orange $(n=10)$ & $1.83-27.5$ & 11.79 & $13.51 \pm 9.95$ & Normal \\
\hline Papaya $(n=4)$ & $7.86-47.14$ & 27.5 & $27.5 \pm 16.36$ & Not normal \\
\hline Guava $(\mathrm{n}=3)$ & $5-14$ & 7 & $8.67 \pm 4.73$ & Normal \\
\hline Salak $(n=3)$ & $1.62-6.96$ & 2.17 & $3.58 \pm 2.94$ & Normal \\
\hline Mango $(n=2)$ & $3-19.29$ & 11.14 & $11.14 \pm 11.51$ & Not applicable \\
\hline Melon $(n=2)$ & $19-27.14$ & 23.07 & $23.07 \pm 5.76$ & Not applicable \\
\hline Apple $(\mathrm{n}=1)$ & 12.14 & 12.143 & 12.14 & Not applicable \\
\hline \multicolumn{5}{|l|}{ Protein (g/day) } \\
\hline \multicolumn{5}{|l|}{ Adults } \\
\hline Salted marine fish $(n=56)$ & $2.14-200$ & 15 & $27.66 \pm 34.91$ & Not normal \\
\hline $\operatorname{Egg}(n=47)$ & $7.86-165$ & 31.43 & $41.12 \pm 30.48$ & Not normal \\
\hline Tofu $(\mathrm{n}=39)$ & $7.86-220$ & 39.29 & $61.65 \pm 55.66$ & Not normal \\
\hline Tempeh $(n=37)$ & $7.14-200$ & 42.86 & $59.86 \pm 53.07$ & Not normal \\
\hline Golden fish $(\mathrm{n}=32)$ & $4.5-142.86$ & 19.29 & $46.02 \pm 43.26$ & Not normal \\
\hline Chicken $(\mathrm{n}=33)$ & $1.67-110$ & 7.86 & $20.15 \pm 23.05$ & Not normal \\
\hline Canned sardine $(n=17)$ & $2.33-70$ & 10 & $13.59 \pm 16.64$ & Not normal \\
\hline Meat $(n=6)$ & $1.17-35$ & 10 & $15.39 \pm 16.01$ & Normal \\
\hline \multicolumn{5}{|l|}{ Teenagers } \\
\hline Salted marine fish $(n=14)$ & $6.43-90$ & 15 & $24.45 \pm 27.43$ & Not normal \\
\hline $\operatorname{Egg}(n=10)$ & $15.71-110$ & 51.07 & $46.36 \pm 27.83$ & Normal \\
\hline Tofu $(n=9)$ & $15-165$ & 31.43 & $61.90 \pm 60.22$ & Normal \\
\hline Tempeh $(\mathrm{n}=9)$ & $14.29-150$ & 50 & $65.87 \pm 53.90$ & Normal \\
\hline Golden fish $)(n=9)$ & $4.5-135$ & 35.71 & $44.48 \pm 39.73$ & Not normal \\
\hline Chicken $(\mathrm{n}=8)$ & $1.67-110$ & 4.58 & $29.21 \pm 40.25$ & Normal \\
\hline Canned sardine $(\mathrm{n}=5)$ & $2.33-70$ & 4.67 & $17.87 \pm 29.31$ & Not normal \\
\hline Meat $(n=2)$ & 35 & 35 & 35 & Not applicable \\
\hline \multicolumn{5}{|l|}{ School aged children } \\
\hline Salted marine fish $(\mathrm{n}=25)$ & $3.21-100$ & 7.50 & $16.26 \pm 21.27$ & Not normal \\
\hline $\operatorname{Egg}(n=19)$ & $11.79-82.5$ & 23.57 & $26.05 \pm 20.54$ & Not normal \\
\hline Tofu $(n=16)$ & $3.93-110$ & 15.71 & $22.61 \pm 2.61$ & Not normal \\
\hline Tempeh $(\mathrm{n}=15)$ & $3.57-100$ & 14.29 & $23.12 \pm 27.51$ & Not normal \\
\hline Chicken $(n=14)$ & $0.92-55$ & 5.89 & $12.95 \pm 14.85$ & Not normal \\
\hline Golden fish $(\mathrm{n}=12)$ & $4.5-71.43$ & 19.29 & $26.91 \pm 25.74$ & Normal \\
\hline Canned sardine $(\mathrm{n}=8)$ & $1.17-10$ & 1.75 & $3.37 \pm 3.16$ & Not normal \\
\hline Meat $(\mathrm{n}=2)$ & $0.58-17.5$ & 9.04 & $9.04 \pm 1.2$ & Not applicable \\
\hline
\end{tabular}

\subsection{Mineral Concentration}

During the survey, not all those identified foodstuffs (Table 2) were available to be collected for mineral analysis. Besides, foodstuffs such as egg, tofu, salted marine fish, tempeh, chicken, sardines, and meat were excluded because they were not direct local origin. Only foodstuffs consumed no less than once a week were included for further analysis. Accordingly, it was only 69 samples (76.7\% of 90 samples to be collected) available for mineral analysis i.e. drinking water $(n=12)$, hulled rice $(n=12)$ and unhulled rice $(n=1)$, vegetables $(n=15$, consisting of 6 samples of cassava leaves, 3 samples of thai squash, 2 samples of spinach, and each one sample of kangkung, stringbean, spinach, and longbean), and soil $(\mathrm{n}=10)$. Of 15 vegetable samples, stringbean was excluded because none of minerals were detected. There were 14 golden fish (common carp, Cyprinus carpio) samples, but fruits had only 5 samples (4 bananas and one papaya). Statistical summary of mineral concentration in drinking water and foodstuffs is presented in Table 3. 
Tabel 3: Statistical summary of mineral concentration in drinking water (mg/L), foodstuffs (mg/kg), and soil (mg/kg)

\begin{tabular}{|c|c|c|c|c|c|}
\hline $\begin{array}{l}\text { Exposure media } \\
\text { and mineral }\end{array}$ & $\mathrm{n}^{a}$ & Range & Median & Mean \pm SD & Distribution \\
\hline Drinking water & 12 & & & & \\
\hline Iron & 12 & $0.22-3.76$ & 1.27 & $1.75 \pm 1.82$ & Not normal \\
\hline Rice, hulled & 12 & & & & \\
\hline Chromium & 3 & $0.15-0.88$ & 0.305 & $0,597 \pm 0,391$ & Normal \\
\hline Iron & 12 & $0,69-5,34$ & 0,75 & $1,394 \pm 1,319$ & Normal \\
\hline Mercury & 2 & $0.02-0.06$ & 0.04 & $0.04 \pm 0.028$ & Not applicable \\
\hline Rice, unhulled & 1 & & & & \\
\hline Chromium & 1 & - & & 0.1 & Not applicable \\
\hline Iron & 1 & - & & 25.73 & Not applicable \\
\hline Manganese & 1 & - & & 44.87 & Not applicable \\
\hline Mercury & 1 & - & & 0.35 & Not applicable \\
\hline Vegetables & 14 & & & & \\
\hline Cassava leaves & 6 & & & & \\
\hline Chromium & 3 & $0.08-0.21$ & 0.12 & $0.12 \pm 0.066$ & Normal \\
\hline Iron & 4 & $22.5-36.73$ & 23.685 & $26.65 \pm 6.743$ & Not normal \\
\hline Manganese & 4 & $51.34-105.91$ & 73.4 & $76.012 \pm 27.176$ & Not normal \\
\hline Mercury & 4 & $0.27-1.88$ & 0.765 & $0.92 \pm 0.685$ & Normal \\
\hline Thai squash & 3 & & & & \\
\hline Chromium & 3 & $6.57-132.05$ & 20.73 & $53.117 \pm 68.724$ & Not normal \\
\hline Iron & 3 & $6.57-132.05$ & 69.31 & $69.31 \pm 88.728$ & Not normal \\
\hline Manganese & 3 & $1.99-5.43$ & 2.01 & $3.143 \pm 1.98$ & Normal \\
\hline Mercury & 1 & - & 0.68 & 0.68 & Not applicable \\
\hline Spinach & 2 & & & & \\
\hline Chromium & 1 & - & 0.23 & 0.23 & Not applicable \\
\hline Iron & 2 & $26.01-59.74$ & 42.875 & $42.875 \pm 23.851$ & Not applicable \\
\hline Manganese & 1 & - & 15.08 & 15.08 & Not applicable \\
\hline Mercury & 2 & $0.05-0.28$ & 0.165 & $0.165 \pm 0.163$ & Not applicable \\
\hline Kangkung & 1 & & & & \\
\hline Arsenic & 1 & - & 0.2 & 0.2 & Not applicable \\
\hline Chromium & 1 & - & 0.2 & 0.2 & Not applicable \\
\hline Iron & 1 & - & 99.99 & 99.99 & Not applicable \\
\hline Manganese & 1 & - & 135.16 & 135.16 & Not applicable \\
\hline Mercury & 1 & - & 3.75 & 3.75 & Not applicable \\
\hline Long bean & 1 & & & & \\
\hline Iron & 1 & - & 8.9 & 8.9 & Not applicable \\
\hline Manganese & 1 & - & 13.93 & 13.93 & Not applicable \\
\hline Mercury & 1 & - & 0.02 & 0.02 & Not applicable \\
\hline Fruits & 5 & & & & \\
\hline Banana & 4 & & & & \\
\hline Iron & 4 & $3.64-5.35$ & 4.69 & $4.59 \pm 0.81$ & Not applicable \\
\hline Manganese & 4 & $1.48-13.86$ & 6.81 & $7.24 \pm 5.27$ & Not applicable \\
\hline Mercury & 3 & $0.06-0.11$ & 0.09 & $0.09 \pm 0.03$ & Not applicable \\
\hline Papaya & 1 & & & & \\
\hline Iron & 1 & - & 9.62 & 9,62 & Not applicable \\
\hline Manganese & 1 & - & 1.29 & 1.29 & Not applicable \\
\hline Mercury & 1 & - & 0.09 & 0.09 & Not applicable \\
\hline Fish, fresh water & 15 & & & & \\
\hline Arsenic & 4 & $0.15-0.26$ & 0.175 & $0.19 \pm 0.05$ & Normal \\
\hline Chromium & 12 & $0.04-2.23$ & 0.14 & $0.135 \pm 0.062$ & Normal \\
\hline Iron & 14 & $7.25-24.5$ & 10.59 & $11.892 \pm 4.362$ & Not normal \\
\hline Manganese & 13 & $2.13-13.36$ & 3.73 & $5.172 \pm 3.194$ & Normal \\
\hline Mercury & 10 & $0.03-0.22$ & 0.12 & $0.13 \pm 0.053$ & Normal \\
\hline Selenium & 11 & $0.24-0.47$ & 0.3 & $0.301 \pm 0.067$ & Not normal \\
\hline Soil & 10 & & & & \\
\hline
\end{tabular}




\begin{tabular}{lrllll}
\hline $\begin{array}{l}\text { Exposure media } \\
\text { and mineral }\end{array}$ & $\mathrm{n}^{a}$ & Range & Median & Mean \pm SD & Distribution \\
\hline Arsenic & 10 & $0.66-5.67$ & 2.3 & $2.504 \pm 1.695$ & Not normal \\
Chromium & 9 & $0.01-2.36$ & 0.34 & $0.518 \pm 0.709$ & Normal \\
Iron & 10 & $1158.34-$ & 1545.74 & $1646.048 \pm$ & Not normal \\
& & 2185.5 & & 303.515 & Not normal \\
Manganese & 10 & $6.38-397.02$ & 39.23 & $106.213 \pm 149.768$ & Normal \\
Mercury & 10 & $0.42-42.84$ & 16.175 & $18.133 \pm 18.674$ & .
\end{tabular}

${ }^{a}$ Number in the raws of environmental exposure media refer to the number of samples, while number in the raws of each mineral refers to the number of detected mineral in the samples.

\subsection{Detection Frequency}

The detection frequency of arsenic, chromium, iron, manganese, mercury, and selenium is presented in Table 4 . It records the detected mineral distribution in 69 total samples of drinking water and foodstuffs.

Table 4: Detection frequency $\left(f_{\mathrm{D}}\right)$ of arsenic, chromium, iron, mercury, manganese, and selenium

\begin{tabular}{lcccccccc}
\hline Mineral & $\begin{array}{c}\text { Drinking } \\
\text { water } \\
(\mathrm{n}=12)\end{array}$ & $\begin{array}{c}\text { Rice } \\
(\mathrm{n}=13)^{a}\end{array}$ & $\begin{array}{c}\text { Vegetable } \\
(\mathrm{n}=15)\end{array}$ & $\begin{array}{c}\text { Fruit } \\
(\mathrm{n}=5)\end{array}$ & $\begin{array}{c}\text { Fish } \\
(\mathrm{n}=14)\end{array}$ & $\begin{array}{c}\text { Soil } \\
(\mathrm{n}=10)\end{array}$ & Total & $f_{\mathrm{D}}(\%)$ \\
\hline Iron & 12 & 13 & 11 & 5 & 14 & 10 & 65 & 94,2 \\
Manganese & 0 & 1 & 10 & 5 & 13 & 10 & 39 & 56.5 \\
Mercury & 0 & 3 & 9 & 4 & 10 & 10 & 36 & 52.2 \\
Chromium & 0 & 4 & 8 & 0 & 12 & 9 & 33 & 47.8 \\
Arsenic & 0 & 0 & 1 & 0 & 4 & 10 & 15 & 21.7 \\
Selenium & 0 & 0 & 0 & 0 & 11 & 0 & 11 & 15.9 \\
\hline
\end{tabular}

${ }^{a}$ Including unhulled rice or gabah.

\subsection{Contamination Level}

Depends on the data distribution, mean or median concentrations of minerals in enviornmental exposure media (Table 3) were used to calculate the mineral CL. Eq. (1) was used to derive health-based RV of mineral in drinking water and foodstuffs using RfD, mean body weight (Table 1) and mean or median consumption rates (Table 2). The following RfD values (mg/kg/day) were used: Arsenic 0.0003 [11], chromium 0.003 [12], iron 0.7 [13], manganese 0.14 [14], mercury 0.0001 (as methyl mercury) [15], and selenium 0.005 [16]. Table 5 presents the measured mineral consentration, healthbased RV, and CL of mineral in each environmental exposure media.

Table 5: Concentration ( $\mathrm{C}, \mathrm{mg} / \mathrm{L}$ in drinking water, $\mathrm{mg} / \mathrm{kg}$ in foodstuffs), health-based reference value ( $\mathrm{RV}, \mathrm{mg} / \mathrm{L}$ for drinking water and $\mathrm{mg} / \mathrm{kg}$ for foodstuffs), and contamination level (CL, unitless) of each mineral in drinking water and foodstuffs

\begin{tabular}{lccc}
\hline Mineral and environmental media & $\mathrm{C}$ & $\mathrm{RV}$ & $\mathrm{CL}$ \\
\hline Arsenic & & & 0.174 \\
Kangkung & 0.20 & 1.146 & 0.225 \\
Fish & 0.19 & 0.845 & 0.46 \\
Soil & 2.3 & $5^{a}$ & \\
Chromium $^{b}$ & & & 0.265 \\
Hulled rice $^{\text {Cassava leaves }}$ & 0.597 & 0.361 & 0.003 \\
Spinach & 0.12 & 5.534 & 0.003 \\
Thai squash & 0.037 & 11.46 & 1.178 \\
Kangkung & 53.12 & 2.816 & 0.003 \\
Fish & 0.2 & 11.46 & 0.003 \\
Soil & 0.135 & 8.447 & 0.064 \\
Iron & 0.518 & $8.1^{c}$ & \\
Drinking water & & & 0.034 \\
Rice, hulled & 1.27 & 37.45 & 0.017
\end{tabular}




\begin{tabular}{|c|c|c|c|}
\hline Mineral and environmental media & $\mathrm{C}$ & $\mathrm{RV}$ & $\mathrm{CL}$ \\
\hline Cassava leaves & 26.65 & 1291.4 & 0.018 \\
\hline Spinach & 35.586 & 2675 & 0.013 \\
\hline Thai squash & 69.31 & 657.02 & 0.105 \\
\hline Kangkung & 99.99 & 2675 & 0.037 \\
\hline Long bean & 8.9 & 870.93 & 0.01 \\
\hline Banana & 4.69 & 2880.8 & 0.002 \\
\hline Papaya & 9.62 & 814.13 & 0.012 \\
\hline Fish & 10.59 & 1971.1 & 0.005 \\
\hline Soil & 1545.74 & $1027.7^{d}$ & 1.5 \\
\hline \multicolumn{4}{|l|}{ Manganese } \\
\hline Cassava leaves & 76.01 & 2582.8 & 0.028 \\
\hline Thai squash & 3.143 & 1314 & 0.002 \\
\hline Spinach & 12.516 & 535 & 0.023 \\
\hline Kangkung & 135.2 & 5350 & 0.025 \\
\hline Long bean & 13.93 & 5350 & 0.003 \\
\hline Banana & 6.81 & 5761.5 & 0.001 \\
\hline Papaya & 1.29 & 1628.3 & $8 \mathrm{E}-4$ \\
\hline Fish & 5.172 & 3942.1 & 0.001 \\
\hline Soil & 39.23 & $19.64^{d}$ & 1.997 \\
\hline \multicolumn{4}{|l|}{ Mercury $^{e}$} \\
\hline Rice, hulled & 0.033 & 0.012 & 2.761 \\
\hline Cassava leaves & 0.764 & 0.1845 & 4.139 \\
\hline Thai squash & 0.564 & 0.0939 & 6.013 \\
\hline Spinach & 0.137 & 0.3821 & 0.358 \\
\hline Kangkung & 3.113 & 0.3821 & 8.145 \\
\hline Long bean & 0.017 & 0.3821 & 0.043 \\
\hline Banana & 0.075 & 0.4115 & 0.182 \\
\hline Papaya & 0.075 & 0.1163 & 0.642 \\
\hline Fish & 0.108 & 0.2816 & 0.383 \\
\hline Soil & 18.133 & $0.03^{d}$ & 604.433 \\
\hline \multicolumn{4}{|l|}{ Selenium } \\
\hline Fish & 0.3 & 14.079 & 0.021 \\
\hline
\end{tabular}

${ }^{a}$ Minimum concentration in unpollutted soil [17]. ${ }^{b}$ Calculated as $\mathrm{Cr}(\mathrm{VI})$ from ratio of $\mathrm{Cr}$ total: $\mathrm{Cr}(\mathrm{III})=100: 84$ [18]. ${ }^{c} \mathrm{As}$ threshold effect level for $\mathrm{Cr}(\mathrm{VI})$ [19]. ${ }^{d}$ Mean value in 12 study sites in Indonesia [20]. ${ }^{e}$ Calculated as $83 \%$ of total $\mathrm{Hg}$ [21].

\section{DISCUSSION}

Field survey and measurement of anthropometric exposure factors and sampling of environmental media were conducted on 8 and 9 June 2016 when fasting month (Ramadan 1437 AH) just started. Unlike adults, teenagers and school aged children were not always available at their homes during the normal survey time because most of them were at schools or at works outside Ciguha. Cosequently, the total subjects were only 101 individuals or $51.1 \%$ response rate. After August 2015, ASGM activities in Gunung Pongkor has been banned, leaving Ciguha as abandoned gold mining site. However, there were still few soil crushings and amalgamations operating in inside closed houses. Some locations of the abandoned ASGM site have been transformed into ponds to keep fish or to wetland farms to grow edible plants or vegetables. High mercury concentration in kangkung grown in this wetland with CL greater than 8 folds its RV (Table 5) indicated that the mercury has been uptaken by edible plant.

\subsection{Anthropometric Exposure Factor Characteristicss}


Table 1 shows typical anthropometric characteristics of rural residents with difficult mobility. As refelcted by high exposure frequency $\left(f_{\mathrm{E}}\right)$ with median value of 363 day/year which is almost completely 365 day/year, Ciguha residents spend mostly at home. Previously, the $f_{\mathrm{E}}$ of residents in less isolated three villages in Ring-1 Antam site (i.e. Bantar Karet, Cisarua, Malasari) was 350 day/year [5], exactly the same as the US-EPA default value [22]. In fact, Antam permits only to go to and exit from Ciguha in only three times daily at 5 to $6 \mathrm{am}, 12$ to $13 \mathrm{pm}$, and 17 to $18 \mathrm{pm}$.

Longer $f_{\mathrm{E}}$ results in higher chronic daily intake (CDI), and high CDI causes high health risk. The high CDI value can be even worsened by lower body weight $\left(W_{\mathrm{B}}\right) . W_{\mathrm{B}}$ of Ciguha residents, with mean value of $53.5 \pm 7.1 \mathrm{~kg}$ and median value of only $52.3 \mathrm{~kg}$ (Table 1), was relatively lower than previously reported from Ring-1 Antam site of 55 kg [5] and in many part of Indonesia [23-26]. $W_{\mathrm{B}}$ of Ciguha residents may indicate typical Sundanse people, a native West Javanese population, who traditionally consume lot of raw, uncooked low caloric vegetables. Accidentally, the survey was conducted in Ramadan where muslim peopple eat only two times daily, although the menu is usually better than in nonfasting month.

Consumption rate is also important as body weight because it directly proportional to the CDI. Although most Ciguha residents are Sundanese, amazingly their daily consumption habit cannot be categorized into high vegetable and fruit consumers. As shown in Table 2, vegetable and fruit consumption among adults ranged from 32 to 76 and from 20 to $46 \mathrm{~g} /$ day, respectively. At national level, mean consumption rates are $57 \mathrm{~g} /$ day vegetables and $34 \mathrm{~g} /$ day fruit [27]. In West Java province, vegetables and fruit consumptions are 47 and $59 \mathrm{~g} /$ day, respectively. These rates are far below the WHO recommmeded values of $400 \mathrm{~g} /$ day fruit and vegetables excluding potatoes and and other starchy tubers [28]. Among Ciguha adult residents, daily vegetable consumption was stringbean $>$ thai squash $>$ cassava leaves $>$ spinach $>$ kangkung $>$ longbean, while fruit consumption was papaya $>$ orange $>$ banana $>$ guava $>$ mango (Table 2 ).

Consumption rate of fish was also low. As shown in Table 2, Ciguha residents consumed more frequent salted marine fish than fresh water fish, but fresh water fish consumption rate was greater, about 1.8 folds that of marine fish. However, current mean consumption rate of fresh water fish (i.e. golden fish) of approximately 45 g/day (Table 2) was only $58 \%$ of the national rate (78 g/day) [27]. This value is not much different with Washington DC default value of 54 g/day [29].

Low fish and fish products consumption may result negatively in low protein and mineral intakes (and so energy requirement), but positively prevent overload exposure to toxic minerals. In polluted water environment, fish can bioaccumulate toxic minerals including heavy metals [30]. In Ciguha, high non-fish protein diet such as tempeh, tofu, and egg were also consumed even in greater rate than fish at 65, 62, and $46 \mathrm{~g} /$ day, respectively. So, in Ciguha low fish consumption might not result in protein and energy deficiency. In developed state such as Washington DC, fish consumption even very low no more than $6.5 \mathrm{~g} /$ day [29].

\subsection{Mineral Distribution}

Table 4 shows that iron is the most distributed mineral that was detected in all environmental exposure matrices. Water has less mineral content because only iron was detected in all water samples. In Ciguha, most water sources are from spring water flowing from non mining site mountain or hills. All residents use this water instead of groundwater.

Mercury was previously predicted to be the most distributed mineral. But, as shown in Table 4, it was only in the $3^{\text {rd }}$ position after iron and manganese with detection frequency more than $50 \%$. Mercury was detected in all soil samples but less in fish $(71.4 \%)$, fruit $(60 \%)$, vegetable $(53.3 \%)$, and rice $(15.4 \%)$. In contrast, iron was detected in all environmental miatrices. Manganese was detected in all soil, fish, and fruit samples, while in vegetable and rice it was detected in 80 and $76.9 \%$ samples, respectively. Arsenic was detected in all soil samples, but only 28.6 and $6.7 \%$ detected in fish and vegetable, respectively. Likely, soil was the critical environmental medium in mercury contamination and distribution.

Mercury precipitate in soil may partly dissolve in water which was further transfered to aquatic biota. After August 2015 when ASGM activity in Gunung Pongkor was stopped, some abandoned amalgamation sites have been tranformed into water ponds to keep fish and plants including rice and water vegetables. Kangkung, an edible water hyacinth grown in wet soil or ponds, contained high level of mercury (see Contamination Level and Load below). Water mineralization, by which metals such mercury are dissolved, is faiclitated by acidic condition. In Gunung Pongkor, surface water is mostly acidic with mean and median $\mathrm{pH}$ of $6,74 \pm 0,47$ and 6.88 , respectively [31]. It is therefore not surprising where mercury was detected in kangkung, thai squash, cassava leaves, and rice with CL of 8.15, 6.01, 4.14, and 2.76 folds, respectively (Table 5).

\subsection{Contamination Level and Load}

Table 5 shows that mercury was the highest contaminant among the six minerals with CL up to more than 8 folds greater that its RV. A contaminant in specified environmental medium with CL>1 means that it has polluted that medium. Accordingly, only mercury and chromium have polluted foodstuffs. Mercury has polluted kangkung, thai squash, cassave 
leaves, and rice by $8.15,6.01,4.14$, and 2.76 folds, respectively, while chromium has only pollutted thai squash by 1.18 folds.

By those CL value (Table 5), pollution status of the six minerals was mercury>chromium> arsenic >iron>manganese>selenium. But, these CL values have different contamination load (C-Load) among the exposure media. For example, the mercury C-Load was kangkung>thai squash> cassava leaves>rice>papaya $>$ fish $>$ banana $>$ longbean, while the iron C-Load was thai squash> kangkung $>$ cassava leaves $>$ rice $>$ papaya $>$ longbean $>$ fish $>$ banana.

Clearly, different plant species has different ability to absorb particular minerals. Aquatic plant including water hyacinth has very high ability to absorb metal ions such as mercury and chromium from aqueous solutions [32]. Wetland plants such as Phragmites australis and Typha latifolia, which can survive in low $\mathrm{pH}$ and high metals environment, are commonly used to absorb aluminium, iron, and manganese in contaminated soil [33]. Kangkung, thai squash, and cassava leaves are likely high mercury accumulator among grown foodstuffs in Ciguha as shown by high CL values.

From health impact point of view, the CL cannot be interpreted solely. Rather, it should be accompanied by assessment of essentiality and toxicity. Contaminant with $\mathrm{CL}<1$ is not automatically safe because it may contribute to adverse health effects due to intake deficiency. This concept is applicable only for minerals having both essentiality and toxicity such as boron, chromium, cobalt, copper, iron, iodine, manganese, molybdenium, nickel, selenium, silicon, vanadium, and zinc [34] (the essentiality and toxicity issues are addressed in the second part of the PHRA report to be published later elsewhere).

Essentiality and toxicity assessment requires exhausted data on a wide variety of consumption rates of foodstuffs. In the present study, fruit and vegetable intake was measured by 24-HDR combined with food frequency and weighted record in two cosecutive days. In the first day, subjects were asked to mention foodstuffs in 4 concecutives meals (yesterday breakfast, yesterday lunch, yesterday dinner, and today breakfast). In practice, during 2-day survey, fruit and vegetable that mentioned in the 24-HDR were not always available to be collected for mineral analysis. Alternatively, fruit and vegetable samples were collected whenever available during 2 days survey, regardless they were mentioned or not in the 24-HDR. Availability of fruit and vegetable in a specified period of time ( 2 days in the present study) actually reveals daily habit of food consumption, so data on food items with quantitative portion size of each fruit and vegetable reported here could be reliable.

However, in the present study the mineral intakes from foods might be underestimate because the available foods are lessen than the mentioned ones. In addition, chromium was not speciated, so it is also less certain whether or not $\mathrm{Cr}$ (III) intake meets its essentiality. Exhausted list of foods or food groups that are certainly consumed in specified amount and frequency is very important to describe mineral distribution in environmental exposure media that contact to human.

In conclusion, among the six minerals analyzed, mercury was the most critical contaminant that has highly polluted soil, kangkung, thai squash, cassava leaves, and rice, whereas chromium has only polluted thai squash. However, the most distributed mineral was iron and the least distributed was selenium, while mercury distribution was only in the third position after iron and manganese. Soil was likely the most and the main polluted environmental medium by mercury from which this contaminant was distributed to grown foodsuffs and water biota. Overall, the order of magnitude of mineral contamination was mercury>chromium>arsenic>iron>manganese >selenium. Meanwhile, anthropometric characteristics of Ciguha residents represented typical population living in isolated rural area of ASGM site with difficult access and limited available local foods.

More extensive food frequency survey, food consumption rate measurement, and food mineral analysis are required to provide a comprehensive data for assessing mineral distribution, contamination, and chronic daily intake. It is necessary also to extend this assessment with additional minerals having physiological and toxicological importance such as boron, cobalt, copper, iodine, molybdenium, nickel, silicon, vanadium, and zinc in more accurate chemical species.

\section{ACKNOWLEDGMENT}

6. The present study was funded by PITTA Grants 2016 of the Directorate of Research and Community Services (DRPM), Universitas Indonesia. The authors wish to thank Head of Health Office of Bogor Regency for facilitating administrative arrangement including research permit, Primary Health Center (Puskesmas) of Nanggung Sub-District for facilitating field data collection, and officials of Bantar Karet village including village secretary for preparing location and the subjects.

\section{REFERENCES}

1. Antam, Annual Report, 2012: Daya Tahan Finansial Mengatasi Tantangan/Financial Endurance Beyond Challenges, in Reportal, P.T. Aneka Tambang TBK (available at www. antam.com), Jakarta, 2012. 
2. Irawan, I., C.E.F. Mumbunan, and A. Ardianto, Community development in the urban area of a developing country a case study of the Antam-Pongkor gold mine, Java Island. Indonesia Mining Engineering, 57(2), pp. 37-41, 2005.

3. Tim Konservasi Pongkor, Laporan Akhir Pendataan Penyebaran Merkuri pada Wilayah Pertambangan di Daerah Pongkor Kabupaten Bogor, Provinsi Jawa Barat, Daftar Isian Pelaksanaan Anggaran (DIPA) No. 0164.0.L/0.2013.0/XII/2006, Pusat Sumber Daya Geologi, Badan Geologi, Departemen Energi dan Sumber Daya Mineral, Bandung, 2006.

4. Yasuda, M., et al., Metal Concentrations of River Water and Sediments in West Java, Indonesia. Bulletin of Environmental Contamination and Toxicology, 87(6), pp. 669-73, 2011.

5. Rahman, A., et al. Health risk estimates from exposure to minerals in artisanal gold mining site in Gunung Pongkor, Bogor, Indonesia. in Full Paper Proceeding of International Trend in Multidisciplinary Academic Research 2014, vol 1, pp 467-490, ISBN 978-969-9948-24-4 (electronic version avaialable at http://www.globalilluminators.org/wpcontent/uploads/2014/ 12/ITMAR-14-390.pdf), 2014.

6. Lwanga, S.K. and S. Lemeshow, Sample size determination in health studies: A practical manual (software version by K C Lun and Peter Chiem, National University of Singapore), World Health Organization, Geneva, 1997.

7. Foucher, E., Modelling Trace Element Uptake by Plants Grown in Contaminated Soils, (Master of Science Thesis) McGill University, Ottawa, Canada, 2010.

8. Watanabe, C., et al., Water intake in an Asian population living in arsenic-contaminated area, Toxicology and Applied Pharmacology, 198, pp. 272-282, 2004.

9. Indonesia, Pedoman Konversi Berat Matang-Mentah, Berat Dapat Dimakan (BDD), dan Resep Makanan Siap Saji dan Jajanan, Badan Penelitian dan Pengembangan Kesehatan, Kementerian Kesehatan RI, Jakarta, 2014.

10. US-EPA, Seminar Publication: Risk Assessment, Management and Communication of Drinking Water Contamination, Office of Drinking Water, US Environmental Protection Agency, Cincinnati OH, 1990.

11. IRIS, Arsenic, inorganic (CASRN 7440-38-2) (electronic version available at http://www.epa. gov/iris/subst/0278.htm), Integrated Risk Information System, US Environmental Protection Agency, 1998.

12. IRIS, Chromium (VI) (CASRN 18540-29-9) (electronic version available at http://www.epa. gov/iris/subst/0144.htm), Integrated Risk Information System, US Environmental Protection Agency, 1998.

13. PPRTV, Provisional Peer Reviewed Toxicity Values for Superfund, Derivation Support Documents (electronic version available at http://hhpprtv.ornl.gov/quickview/pprtv_papers.php), Integrated Risk Information System, US Environmental Protection Agency, 2014.

14. IRIS, Manganese (CASRN 7439-96-5) (electronic version available at http://www.epa.gov/ iris/subst/0373.htm), Integrated Risk Information System, US Environmental Protection Agency, 2001.

15. IRIS, Methylmercury (MeHg) (CASRN 22967-92-6) (electronic version available at http://www. epa.gov/iris/subst/0073.htm), Integrated Risk Information System, US Environmental Protection Agency, 1987.

16. IRIS, Selenium and Compounds (CASRN 7782-49-2) (electronic article retrieved on 29 June 2015 from http://www.epa.gov/iris/subst/0472.htm), Integrated Risk Information System, US Environmental Protection Agency, 1991.

17. Ferguson, J.E., The Heavy Elements: Chemistry, Environmental Impact and Health Effect, Oxford, Pergamon Press, 1990.

18. Soarea, M.E., E. Vieira, and M.d.L. Bastos, Chromium Speciation Analysis in Bread Samples. J. Agric. Food Chem., 58, pp. 1366-1370, 2010.

19. Hubner, R., K.B. Astin, and R.J.H. Herbert, Comparison of sediment quality guidelines (SQGs) for the assessment of metal contamination in marine and estuarine environments, J. Environ. Monit., 11, pp. 713-722, 2009.

20. Suhariyono, G. and Y. Menry. Analisis karakteristik unsur-unsur dalam tanah di berbagai lokasi dengan menggunakan XRF, in Prosiding PPI-PDIPTN 2005, Puslitbang Teknologi Maju-BATAN, Yogyakarta, 2005.

21. Hajeb, P., S. Jinap, and I. Ahmad, Biomagnifications of mercury and methylmercury in tuna and mackerel, J. Environ. Monit. Assess., 171, pp. 205-217, 2010.

22. US-EPA, Exposure Factors Handbook: 2009 Update. External Review Draft July 2009, EPA/600/R-09/052A, Office of Research and Development, National Center for Environmental Assessment, US Environmental Protection Agency, Washington DC, 2009.

23. Suryaman, U.S. and A. Rahman, Safe Area for Residential Population to Reside Near Limestone Mining: A Risk Management Approach, J. Ekolog. Kesehatan, 10(4), pp. 256-265, 2011.

24. Rahman, A., et al., Health risk estimates from exposure to pulp and paper mills contaminants, in Proceeding of the International Federation of International Health $12^{\text {th }}$ World Congress ISBN 978-88-7587-664-7, Medimon S. R. L., Bologna, Italy, 2012.

25. Rahman, A., et al., Analisis Risiko Kesehatan Lingkungan Pertambangan Kapur di Sukabumi, Cirebon, Tegal, Jepara dan Tulung Agung (Environmental Health Risk Assessment of Limestone Mining in Sukabumi, Cirebon, Tegal, Jepara, and Tulung Agung), J. Ekolog. Kesehatan, 7(1), pp. 665-677, 2008.

26. Nukman, A., et al., Analisis dan Manajemen Risiko Kesehatan Pencemaran Udara: Studi Kasus di Sembilan Kota Besar Padat Transportasi, J. Ekolog. Kesehatan, 4(2), pp. 270-289, 2005. 
27. Trihono, et al., eds. Buku Survei Konsumsi Makanan Individual dalam Studi Diet Total 2014, Lembaga Penerbitan, Badan Penelitian dan Pengembangan Kesehatan, Kementerian Kesehatan RI, Jakarta, 2014.

28. WHO, Promoting fruit and vegetable consumption around the world. Global Strategy on Diet, Physical Activity and Health (electronic article retrieved on 3 January 2017 from http://www. who.int/dietphysicalactivity/fruit/en/), 2017.

29. Department of Ecology State of Washington, Fish Consumption Rates, Technical Support Document: A Review of Data and Information about Fish Consumption in Washington, Version 2.0 Final, ed. P.N. 12-09-058, Toxics Cleanup Program, Washington State Department of Ecology, Olympia WA, 2013.

30. Ambreen, F., M. Javed, and B. Ummara, Tissue Specific Heavy Metals Uptake in Economically Important Fish Cyprinus carpio at Acute Exposure of Metals Mixtures. Pakistan Journal of Zoology, 47(2), 2015.

31. Rahman, A., et al., Kandungan logam, metaloida, dan nonlogam dan profil kesehatan masayrakat di wilayah peruntukan pertambangan emas Gunung Pongkor dan wilayah pembanding di Pamijahan, Kapupaten Bogor, Pusat Teknologi Intervensi Kesehatan Masayarakat, Badan Penelitian dan Pengembangan Kesehatan, Kementerian Kesehatan RI (unpublished work), Jakarta, 2013.

32. Haider, S.Z., et al. in Proceeding of International Conference on Water Hyacinth, United Nation Environment Programme, Nairobi, Kenya, 1984.

33. Guo, L. and T.J. Cutright, Remediation of acid mine drainage (AMD)-contaminated soil by Phragmites australis and rhizosphere bacteria, Environmental Science and Pollution Research International, 21(12), pp. 7350-60, 2014.

34. National Academy of Sciences, Dietary Reference Intakes for Vitamin A, Vitamin K, Arsenic, Boron, Chromium, Copper, Iodine, Iron, Manganese, Molybdenum, Nickel, Silicon, Vanadium, and Zinc (electronic version retrieved on 1 September 2015 from http://www.nap.edu/ catalog/10026.htm), 2001. 